\title{
Digital- und Medienpolitik in Parteiprogrammen zur Bundestagswahl 2021
}

\author{
Vier Parteien - Die Linke, SPD, Bündnis 90/Die Grünen und FDP - haben bis Ende April 2021 \\ den Entwurf ihres Programms zur Bundestagswahl am 26. September veröffentlicht. Wie \\ klar und überzeugend vermitteln Bundestagsparteien ihre Vorstellungen? Die digital- und \\ medienpolitischen Aussagen der Entwürfe werden kritisch analysiert.
}

Die Bundestagswahl wirft ihren Schatten voraus. Die Führungen der politischen Parteien haben seit Februar 2021 Programmentwürfe zu dieser Wahl veröffentlicht, um offenzulegen, was sie in der 20. Legislaturperiode erreichen wollen. Am 30.4.2021 waren die Entwürfe der Linken (2021), SPD (2021), Grünen (2021) und FDP (2021) im Netz allgemein zugänglich. In den Programmen wird einhellig die große Bedeutung der Digitalisierung sämtlicher Bereiche der Gesellschaft und speziell des Mediensektors für die Entwicklung Deutschlands betont. Angesichts dieser Akzentsetzung bietet die nachfolgende Analyse eine kurze vergleichende kritische Auseinandersetzung mit Aussagen der Programmentwürfe auf den drei Feldern öffentlich-rechtlicher Rundfunk (ÖRR) und private Massenmedien, digitale Plattformkonzerne sowie Ausbau von Netzen für die elektronische Kommunikation. Innerhalb dieser Felder werden die Absichtserklärungen der Parteien nach der zeitlichen Reihenfolge ihrer Veröffentlichung geordnet.

Öffentlich-rechtlicher Rundfunk und private Massenmedien

Die Linke

Die Linke hält den ÖRR ,als Mediengrundversorgung für alle [für] unentbehrlich“ (Die Linke, 2021, 139). Die Partei

(C) Der/die Autor:in(nen) 2021. Open Access: Dieser Artikel wird unter der Creative Commons Namensnennung 4.0 International Lizenz veröffentlicht (creativecommons.org/licenses/by/4.0/deed.de).

Open Access wird durch die ZBW - Leibniz-Informationszentrum Wirtschaft gefördert

Prof. Dr. Torsten J. Gerpott leitet den Lehrstuhl für Unternehmens- und Technologieplanung an der Mercator School of Management der Universität Duisburg-Essen. fordert, dass dessen Programme „als gemeinsame Plattformen auftreten und ... nicht kommerzialisiert werden dürfen“ und ihr „Empfang ... bezahlbar bleiben“ muss (Die Linke, 2021, 139). ÖRR-Sender sollten „technisch auf dem Stand veränderter Mediennutzungen" (Die Linke, 2021, 139), „transparent“ und ihre Eigenproduktionen „zeitlich unbegrenzt in den Mediatheken abrufbar sein“ (Die Linke, 2021, 140). Teile des Rundfunkbeitrags will man verwenden, „um nicht kommerzielle Angebote, die einen Beitrag zur öffentlichen Meinungsbildung, Kultur, Bildung und Information leisten, zu finanzieren" (Die Linke, 2021, 140). „Journalist*innen in den privaten Printmedien“ will die Linke über eine „Kontrolle durch die Öffentlichkeit“ (Die Linke, 2021, 139), eine Reform des Urhebervertragsrechts zu ihren Gunsten sowie die Abschaffung des Leistungsschutzrechts der EU zur Seite stehen, ohne aber „das Recht auf Nutzung“ (Die Linke, 2021, 139) abzuschwächen. Alles in allem zielen die zum Teil in sich widersprüchlichen Vorstellungen der Linken auf eine (noch stärkere) Vergesellschaftung des ÖRR-Systems und eine wirtschaftliche sowie publizistische Schwächung privatwirtschaftlicher Medienunternehmen.

SPD

Die SPD setzt sich „gesamtstaatlich für einen starken ÖRR ein“ und will „die Länder darin [unterstützen], den Auftrag in einer digitalen Medienwelt weiter zu entwickeln“ (SPD, 2021, 47), um „eine umfassende und tiefgreifende journalistische Berichterstattung sicher[zu] stellen" (SPD, 2021, 13). Damit wird verbrämt, jegliche Ausweitung von ÖRR-Angeboten im Internet gutzuheiBen. Dies wird auch dadurch erkennbar, dass die Partei „Qualitätsinhalte der ÖRR-Sender Europas“ (SPD, 2021, 13) jedem mittels „eine[r] europäische[n] Medienplattform ... grenzüberschreitend zugänglich“ (SPD, 2021, 13) machen möchte. Gleichzeitig verspricht die SPD aber, „Verlagen ... dabei [zu] helfen, die Transformation ins Digitale erfolgreich zu bewältigen“ und hierzu „die Rahmenbedingungen privatwirtschaftlichen Medienschaffens“ auf 
den Feldern „Wettbewerbs-, Urheber- oder Telekommunikationsrecht" (SPD, 2021, 47) zu stärken sowie die oben aufgeführte europäische Medienplattform „auch für private Qualitätsanbieter aus Medien und Kultur zugänglich“ (SPD, 2021, 13-14) zu machen. Weder erfährt man Konkreteres zu den rechtlichen Maßnahmen, noch äußern sich die Sozialdemokraten gehaltvoll dazu, wie sie sich im Spannungsfeld zwischen staatsnahen öffentlichen und privaten Medienanbietern positionieren wollen. Insgesamt verströmt das SPD-Programm einen altmodischen besserwisserischen Geist, der Massenmedien primär als staatsnahes pädagogisches Mittel zur Verbreitung von Qualitätsinhalten versteht ohne zu erklären, wer mit welcher Legitimation über den „Wert“ von Inhalten entscheiden soll.

\section{Bündnis 90/Die Grünen}

Die Grünen wollen, dass die öffentlich-rechtlichen Rundfunksender „stark und zukunftsfest" aufgestellt werden, „alle gesellschaftlichen Bereiche" abdecken und eine „ausreichende Finanzierung“ (Bündnis 90/Die Grünen, $2021,95)$ erhalten. Deren gegenwärtig fragmentierte Mediatheken will die Partei „zu gemeinsamen Plattformen weiterentwickeln“ (Bündnis 90/Die Grünen, 2021, 95), die mit einer „werbefreie[n], offen[en] und multilingual[en] europäische[n] digitale[n] Plattform in öffentlicher Hand“ (Bündnis 90/Die Grünen, 2021, 114) verzahnt werden sollen. Damit sich die deutschen Sender aber nicht zu entspannt zurücklehnen und um dem derzeit allgemein spürbaren Unbehagen an deren schwachem Reformwillen im Hinblick auf Effizienz sowie Angebotsbreite und -tiefe bei der herkömmlichen linearen sowie der Verbreitung über das Internet Rechnung zu tragen (etwa Buhrow, 2021; Hartung, 2021), möchten die Grünen gleichzeitig „eine Debatte darüber führen, wie öffentlich-rechtliche Medien im 21. Jahrhundert aussehen sollen“ (Bündnis 90/ Die Grünen, 2021, 95). Dazu, welche Konsequenzen mit der Debatte angestrebt werden, äußert man sich jedoch nicht. Ebenso widmet das Programm der Frage kein Wort, wie für private Presseverlage und Rundfunkveranstalter gegenüber unverändert großzügig finanzierten öffentlich-rechtlichen Sendern mit ausgeweiteter Netzpräsenz faire Wettbewerbschancen geschaffen werden sollen. Die Geringschätzung privater Anbieter von Publikumsmedien zeigt sich darin, dass die Grünen zwar „bei kulturellen Werken ... für Urheber*innen eine angemessene Vergütung“ (Bündnis 90/Die Grünen, 2021, 109) sichern wollen. Nicht erörtert wird, ob zu solchen Werken auch Videoclips der Fußballbundesligen oder kurze Pressetexte von Unternehmen gehören sollen, die als Bagatellnutzungen gemäß $\S 10$ des Entwurfs der Bundesregierung vom 9.3.2021 für ein kurz vor dem Inkrafttreten stehendes Urheberrechts-Diensteanbieter-Gesetz demnächst bis zu
15 Sekunden bzw. 160 Zeichen durch jeden im Netz nicht kommerziell verbreitet werden dürfen (Bundesregierung, 2021b). Alles in allem fehlen in dem Programm der Grünen bezüglich ihrer Pläne für den ÖRR und privatwirtschaftlichen Mediensektor inhaltlich eindeutige Konturen.

\section{FDP}

Die FDP plädiert für eine „Auftrags- und Strukturreform“ des ÖRR, der sich sowohl bei der Verbreitung seiner Programme auf den klassischen Wegen als auch im Internet „primär auf Nachrichten, Kultur, politische Bildung und Dokumentationen konzentrieren soll" (FDP, 2021, 41). Mit dieser Ausrichtung wollen die Liberalen die Wettbewerbschancen „privater Presse- und Medienhäuser" sowie „die Medien- und Meinungsvielfalt stärken“ (FDP, 2021, 42). Die Stärkung soll durch ein Urheberrecht flankiert werden, das dazu beiträgt, „dass geistige Werke leicht zugänglich“ bleiben, aber dennoch „Erträge aus der Verwertung kreativer Leistungen ... den Urhebern und den weiteren Berechtigten zufließen“ (FDP, 2021, 48). Das soll im Netz „durch eine Bagatellklausel für private Nutzungen“ (FDP, 2021, 48) erreicht werden. Inwiefern die Gestaltung einer solchen Klausel in dem eben erwähnten Gesetzentwurf der Bundesregierung den Vorstellungen der FDP entspricht, ist nicht zu erkennen. Klar, aber ohne jegliche Begründung, sprechen sich die Liberalen indes gegen ein „Leistungsschutzrecht für Presseverleger" (FDP, 2021, 48) aus (allgemein zu diesem Recht: Specht-Riemenschneider, Riemenschneider und Schneider, 2020, 76-77).

\section{Zwischenfazit}

In einer Gesamtschau ähneln sich die Positionen der Linken, SPD und Grünen im Hinblick auf das ÖRR-System sehr stark. Die drei Parteien stellen es nicht ansatzweise infrage und wollen es online stärken. Kritische Töne bezüglich der Systemeffizienz sind bei innen kaum zu vernehmen. Privaten Anbietern von Publikumsmedien begegnen sie mit Skepsis, was sich unter anderem in Unverständnis für Maßnahmen zur Verbesserung urheberrechtlicher Positionen solcher Unternehmen widerspiegelt. Zu diesen Wertungen setzt die FDP einen durch Vertrauen in marktwirtschaftliche Prozesse und privatautonomes Handeln geprägten Kontrapunkt.

\section{Digitale Plattformkonzerne}

\section{Die Linke}

Die Linke will die wirtschaftliche und politische „Macht der Internetkonzerne und Plattformen“, von denen sie explizit „Google, Amazon, Facebook, Apple und Microsoft" 
(Die Linke, 2021, 89) nennt, durch Zerschlagung der Unternehmen begrenzen. An deren Stelle sollen „commonsbasierte öffentliche Alternativen“, „Plattformgenossenschaften und öffentlich-rechtlich betriebene Plattformen“ (Die Linke, 2021, 90) sowie Dorfläden „mit Zugang zu Bestellplattformen der regionalen Produzenten" (Die Linke, $2021,80)$ gefördert werden. Was man mit diesen Schlagworten und mit Förderung meint, wird nicht verdeutlicht. Ebenso irritiert, dass die Linke seit langem belegte und schon in Einführungslehrbüchern erklärte Größen- und Verbundvorteile in der Plattformökonomie sowie die sich aus innen ergebenden Tendenzen zur Marktkonzentration auf wenige Anbieter oder gar zum Kippen in Richtung eines Monopols (etwa Clement et al., 2019, 238-257; Monopolkommission, 2015, 35-39 und 129-131) ignoriert. Eine solche Programmatik ist bestenfalls naiv und schlimmstenfalls anmaßend. Sie lässt die Verschwendung von Steuergeldern für Dezentralisierungs- und Kooperationsideologien befürchten.

Die Partei strebt ein „Plattformstrukturgesetz“ und für Wettbewerber "ein Zugriffsrecht auf Daten von Plattformen .., die auf Datenmonopolen basieren" (Die Linke, 2021, 90), an. Die Linke hinkt mit diesen Forderungen dem Status quo hinterher. Auf der EU-Ebene beinhaltet der Vorschlag der Europäischen Kommission (2020b) für einen Digital Markets Act (DMA) Möglichkeiten für Kartellbehörden, ökonomisch bedeutsame Plattformkonzerne aufzuspalten (Art. 16 Abs. 1 DMA) und innen vorbeugend die Verpflichtung, Wettbewerbern Datenzugang zu gewähren (Art. 6, Abs. 1, lit. i DMA), aufzuerlegen (Gerpott, 2021a; Krämer und Schnurr, 2021; Polley und Konrad, 2021; Seip und Berberich, 2021). Äquivalente Interventionsoptionen wurden auf nationaler Ebene in Deutschland mit der am 19.1.2021 in Kraft getretenen 10. Novelle des Gesetzes gegen Wettbewerbsbeschränkungen (GWB) für das Bundeskartellamt im Hinblick auf strukturelle Eingriffe (§ 32 Abs. 2 GWB) und Vorfeldverbote beim Datenzugang für Wettbewerber großer Plattformbetreiber (§ 19, Abs. 2 Nr. 4, § 19a, Abs. 4 und $\S$ 20 Abs. 1a GWB) geschaffen (Gerpott, 2021b; Gerpott und Mikolas, 2021; Lettl, 2021; Nagel und Hillmer, 2021; Schweda und Schreitter, 2021).

Bei der Menge der über große digitale Plattformen verbreiteten Inhalte liegt es nicht fern, dass deren Betreiber illegalen Uploads nicht von Hand, sondern nur unter Einsatz intelligenter Algorithmen weitgehend maschinell automatisiert im Netz entgegentreten können (Gerpott 2019, 422). Dennoch lehnt die Linke Upload-Filter zum Schutz von Urheber- und Persönlichkeitsrechten vor allem auf Plattformen zum Teilen von Inhalten ebenso ab wie die - derzeit in § 3 Abs. 2 Netzwerkdurchsetzungsgesetz verankerten Regelungen zur - Sperrung von In- halten durch Betreiber sozialer Netzwerke ohne richterlichen Beschluss. Diese Sicht ist populär. Sie klärt aber nicht, wie die Partei Persönlichkeits- und Urheberrechtsschutz im Netz trotzdem angemessen vorantreiben will. Zwar fordert die Linke die strafrechtliche Verfolgung von „Hasskriminalität gegen queere Menschen, Communities oder ihre Unterstützer*innen“ (Die Linke, 2021, 104). Darüber hinaus gehende differenzierte Überlegungen zum Umgang mit Hasskriminalität auf digitalen Plattformen sucht man in ihrem Programm vergeblich. Netzneutralität soll "grundgesetzlich gesichert werden“ (91). Warum der bestehende Schutz der Netzneutralität in der Verordnung (EU) 2015/2120 nicht ausreichen könnte, erklärt die Partei nicht. Insgesamt zeichnen sich die Ausführungen zu großen Betreibern digitaler Plattformen im Programm der Linken durch einen feindseligaggressiven Ton und ein Ausblenden der wissenschaftlichen Fachdiskussion aus.

\section{SPD}

Die SPD verspricht für Betreiber großer digitaler Plattformen "gemeinsam mit den EU-Mitgliedstaaten eine starke und präzise Regulierung" zu schaffen, die gesetzliche Interoperabilitätsverpflichtungen für Messenger-Dienste, soziale Netzwerke und digitale Plattformen sowie eine Integration von „ex-ante-Kontrollen“ (SPD, 2021, 14) in das Kartellrecht beinhalten soll. Große Digitalkonzerne möchte die Partei „durch neue europäische Instrumente ... zähmen oder notfalls .. entflechten" (SPD, 2021, 14). Auch bei der SPD bleibt unverständlich, warum sie nicht zu einschlägigen aktuellen Gesetzesänderungen oder -initiativen zur Interoperabilität von Plattformen (§ 19a Abs. $2 \mathrm{Nr}$. 5 GWB, Art. 6 Abs. 1 lit. f DMA) und zur Begrenzung inrer Marktmacht (vor allem $\S 19$ a und 20 GWB, Art. 5 und 6 DMA) Position bezieht, sondern sie außer Acht lässt (Gerpott, 2021b; Lettl, 2021).

Ebenso wie die Linken wünschen sich die Sozialdemokraten, dass „es ... Alternativen zu den großen Plattformen ... mit echten Chancen für lokale Anbieter" (SPD, 2021, 14) gibt. Sie wollen deshalb „Plattformen für den regionalen Handel und regionale Dienstleistungen fördern" (SPD, 2021, 14). Die zuvor beschriebenen ökonomischen Mechanismen auf Online-Vermittlungsmärkten werden damit verkannt oder als durch politischen Willen überwindbar interpretiert. Die Finanzierung der angekündigten Förderung bleibt im Dunkeln.

Hasskriminalität und anderen Straftaten im Internet will die SPD begegnen, indem sie Plattformbetreibern auferlegt, „Voraussetzungen für eine grundsätzliche Identifizierbarkeit" (SPD, 2021, 14) von Verdächtigen zu schaffen, „die nationalen Schutzvorschriften im Strafgesetzbuch 
und Netzwerkdurchsetzungsgesetz" weiterentwickelt und „verbindliche Regelungen auf europäischer Ebene (Digital Service Act)“ (SPD, 2021, 15) sowie „europäische Frühwarnsysteme gegen Desinformationskampagnen“ (SPD, $2021,53)$ vorantreibt. Abwägungen zwischen dem Schutz der Persönlichkeit sowie der Rede- und Informationsfreiheit im Netz sind bei den Sozialdemokraten ebenso wenig zu finden wie Anhaltspunkte zur praktischen Umsetzung des erwähnten Frühwarnsystems.

Alles in allem formuliert die SPD ihre Vorstellungen zum Umgang mit digitalen Plattformkonzernen etwas weniger aggressiv und klarer als die Linken. Inhaltlich liegen die beiden Parteien auf diesem digitalpolitischen Feld jedoch sehr nah beieinander.

\section{Bündnis 90/Die Grünen}

Auch die Grünen beabsichtigen, „Dienstleistungen von Plattformen... und ... [deren ] Marktmacht [zu] regulieren“ (Bündnis 90/Die Grünen, 2021, 38). Sie äußern sich dazu materiell allerdings eher nebulös. Eine Positionierung zum DMA, der in Art. 5 und 6 lange Listen einschlägiger Verhaltensauflagen enthält, sucht man im Programm vergeblich. Einerseits soll in Deutschland das Bundeskartellamt zukünftig bei „Internetgiganten ... Erwerbsvorgänge“ (Bündnis 90/Die Grünen, 2021, 40) prüfen. Andererseits befürwortet die Ökopartei ein eigenständiges europäisches Kartellamt, um eine „europäische Digitalaufsicht [zu] etablieren" (Bündnis 90/Die Grünen, 2021, 40). Wie eine parallele Kompetenzerweiterung des Bundeskartellamts und eines europäischen Kartellamts harmonieren soll, bleibt im Dunkeln. Ebenso lässt die Partei offen, ob Entscheidungen zur geforderten Option der Aufspaltung von großen Digitalkonzernen „unabhängig von einem Missbrauch ..., wenn ihre Marktmacht zu groß wird" (Bündnis 90/Die Grünen, 2021, 41), vom Bundeskartellamt oder der Kommission gefällt werden sollten. In jedem Fall planen die Grünen die Unterstützung von „Smart-City-Projekte[n zum] Aufbau unabhängiger digitaler Plattformen, mit denen der örtliche Einzelhandel attraktivere Angebote machen kann" (Bündnis 90/ Die Grünen, 2021, 73). Dies deutet auf den Einsatz von Steuergeldern für wirtschaftspolitisch ineffiziente Förderprogramme hin.

Bei der Bekämpfung von „Hasskriminalität im Netz“ (Bündnis 90/Die Grünen, 2021, 96) vertrauen die Grünen vor allem auf eine „zügige Umsetzung des europäischen Digital Services Act“ (Bündnis 90/Die Grünen, 2021, 96; DSA). Dieser EU-Verordnungsentwurf (Europäische Kommission, 2020a) verlagert die Kontrolle und Rechtsdurchsetzung gegenüber großen Plattformen im Zusammenhang mit der Verbreitung illegaler Inhalte auf die
Europäische Kommission, die dabei durch ein neu einzurichtendes Beratungsgremium sowie einen Ausschuss für digitale Dienste unterstützt werden soll (insbesondere Art. 47 Abs. 2 lit. b, 48 Abs. 4 und Art. 70 DSA; Gerpott, 2021a; Spindler, 2021). Das blendet das Programm aus, wenn es parallel für Deutschland im gleichen Absatz zusätzlich zu den bereits vorhandenen Landesmedienanstalten ohne Nutzen-Kosten-Erwägungen eine „gemeinsame Medienanstalt der Länder" (Bündnis 90/Die Grünen, 2021, 96) fordert. Hier hatte man nicht den Mut, den hintergründig mitschwingenden Zweifel an der Wirtschaftlichkeit der heutigen Organisation mit 14 Anstalten pointierter auszudrücken. Die Grünen akzeptieren „eine Verpflichtung zum Einsatz von Uploadfiltern“ (Bündnis 90/Die Grünen, 2021, 96) ebenfalls nicht. Nicht erörtert wird damit, wie die Partei Persönlichkeits- und Urheberrechtsschutz sowie Rede- und Informationsfreiheit austarieren will.

In der Summe hinterlässt das grüne Programm zu digitalen Plattformkonzernen einen ambivalenten Eindruck: Einerseits findet man Hinweise auf nuancierte Kenntnisse der einschlägigen Fachdebatten. Andererseits mangelt es an präzisen, in sich widerspruchsfreien wirtschaftspolitischen Vorschlägen zu dieser Anbietergruppe.

\section{FDP}

Die Liberalen fordern, dass Digitalkonzerne „einer speziellen Regulierung unterworfen werden“, um zu verhindern, dass sie „den Wettbewerb verzerren“ (FDP, $2021,8)$. Staatseingriffe sollen nicht allein auf nationaler, sondern zudem auf EU-Ebene erfolgen. Deshalb unterstützt die Partei „die Pläne zur Schaffung eines Digital Markets Act“ (FDP, 2021, 8), allerdings ohne zu skizzieren, wie man sich die Verzahnung der zwei Ebenen vorstellt. „Den Einsatz von Uploadfiltern im Netz" lehnen die Liberalen „als immense Gefahr für Meinungs- und Kunstfreiheit ... ab“ (FDP, 2021, 48). „Meinungsfreiheit in vollem Umfang“ (FDP, 2021, 41) will die Partei zudem unter anderem durch eine Abschaffung des Netzwerkdurchsetzungsgesetzes und einer damit verbundenen Beseitigung von Sperrungsentscheidungen privater Unternehmen, die man „durch Einrichtungen der Selbstregulierung als Beschwerdeinstanz" (FDP, 2021, 41) ersetzen möchte, gewährleisten. Was man sich unter solchen Einrichtungen vorstellen darf, bleibt verborgen. Opfern von Hassreden oder anderen Vergehen im Netz soll mit einem „Auskunftsanspruch gegen Plattformen und Internetprovider" (FDP, 2021, 41) geholfen werden. Netzneutralität will die Partei schützen und „neue qualitätsgesicherte Dienste ermöglichen“ (FDP, 2021, 26). Auch bei der FDP bleibt unklar, warum hier über die Verordnung (EU) 2015/2120 hinaus weitere staatliche Maßnahmen geboten sein könnten. 
Insgesamt nimmt das Programm der FDP wirtschaftspolitisch verhältnismäßig genau konturierte Positionen gegenüber digitalen Plattformkonzernen ein.

\section{Zwischenfazit}

Die Programme der Linken und der SPD greifen aktuelle rechtliche Entwicklungen zum Umgang mit Betreibern großer Online-Plattformen nicht auf und vermitteln den aus wirtschaftswissenschaftlicher Sicht befremdlichen Eindruck, dass durch politische Maßnahmen die Wettbewerbsbedeutung lokaler Plattformen effizient gesteigert werden könne. Das Programm der Grünen ist in sich nicht widerspruchsfrei und inhaltlich sehr offen gehalten. Die Vorstellungen der FDP weichen oft deutlich von denen der anderen drei Parteien ab.

\section{Netzausbau für die elektronische Kommunikation}

\section{Die Linke}

Die Partei kündigt an ,mit einem öffentlichen Investitionsprogramm die enormen Mängel bei[m] Breitbandausbau [zu] beheben“ (Die Linke, 2021, 11). Das Programm soll sich auf „10 Mrd. Euro jährlich in ganz Deutschland“ (Die Linke, 2021, 90) belaufen. Den „Vorrang privater Anbieter sowohl beim Ausbau als auch beim Betrieb der regionalen Breitbandnetze“ (Die Linke, 2021, 79) will die Linke abschaffen. Breitband- und Mobilfunknetze sowie die Deutsche Telekom möchte man „in öffentliche (oder genossenschaftliche) Hand und in gesellschaftliche Eigentumsformen“ (Die Linke, 2021, 59) überführen. Es soll nur noch „ein einheitliches Mobilfunknetz aus einer Hand“ (Die Linke, 2021, 90) geben. Die Partei fordert, sämtliche Netze „durch die Kommunen ... dauerhaft in öffentlicher Hand [zu] betreiben“ (Die Linke, 2021, 90). Allein schon aus technischen Gründen würde eine Umsetzung dieser Forderung zumindest für Mobilfunk- und überregionale leitungsgebundene Transportnetze zu ineffizienten Lösungen führen. Außerdem verzichten die Linken auf einen konkreten Vorschlag, wie die Enteignungen finanziert werden und ablaufen sollen.

Alles in allem plädiert die Linke beim Bau und Betrieb von Telekommunikationsnetzen unmissverständlich für eine staatsmonopolistisch-sozialistische Ordnungspolitik.

\section{SPD}

Die SPD verspricht „die Versorgung aller Haushalte und Unternehmen mit Bandbreite von mindestens $1 \mathrm{Gbit} / \mathrm{s}$ [zu] garantieren - durch konkrete, gesetzlich festgelegte Ausbau- und Versorgungsverpflichtungen und entsprechende Zwischenziele" (SPD, 2021, 12-13). Wie diese Garantie fi- nanziell und praktisch umgesetzt werden könnte, wird weder durch den Hinweis, dass ,hier .. auch die Netzbetreiber in der Verantwortung [stehen]" (SPD, 2021, 13) noch durch andere Überlegungen erkennbar. In der Summe konvergiert die inhaltliche Substanz des Programms im Hinblick auf den Telekommunikationssektor gegen Null.

\section{Bündnis 90/Die Grünen}

Die Grünen nennen aktuelle Fachthemen zum Netzausbau umfassender als die drei politischen Konkurrenten. So werden die Gestaltung eines Breitband-Universaldienstes zur Absicherung eines persönlichen Rechtsanspruchs auf einen hinreichend schnellen Internetzugang zur privaten Nutzung in der Wohnung und unterwegs, die Liefertreue bei der Übertragungsgeschwindigkeit von Internetanschlüssen, lokales Roaming von Mobilfunknetzbetreibern und die Beibehaltung von Versteigerungen als vorrangiges Verfahren bei der Vergabe von Frequenzen erwähnt (Bündnis 90/Die Grünen, 2021, 72-74). Die Vorstellungen bleiben zumeist sehr allgemein (z. B. Mindestqualität der Breitbandversorgung, Abruf von Fördergeldern des Bundes zum Netzausbau). Außerdem werden sie nicht mit der zum Zeitpunkt der Programmveröffentlichung (19.3.2021) bereits im Detail erkennbaren Novelle des Telekommunikationsgesetzes (TKG; Bundesregierung, 2021a) verknüpft (z.B. Universaldienst in §§ 156157 TKG 2021, Liefertreue in §§ 57-58 TKG 2021, Roaming in § 106 TKG 2021; Deutscher Bundestag, 2021) und sind folglich nicht à jour. Finanzierungsfragen werden nicht angesprochen.

\section{FDP}

Die FDP fordert „eine flächendeckende und hochleistungsfähige Mobilfunkabdeckung durch echten Wettbewerb auf dem Mobilfunkmarkt, ein Glasfasernetz und eine konsequente Hochrüstung bestehender 4G Netze“ (FDP, 2021, 25). Der Netzausbau soll „,mit einem effizienten Auktionsdesign und durch ein starkes und zeitnahes Controlling des Bundes“ sowie „nachfrageorientiert und kosteneffizient ... durch Gigabit-Gutscheine für Privathaushalte und kleine und mittlere Unternehmen“ (FDP, 2021, 25) erreicht werden. Starke Argumente gegen derartige Gutscheine (Gerpott, 2020) werden nicht gewürdigt. Im Mobilfunk greifen die Liberalen technische Trends auf, indem sie „Spezialdienste mit Network-Slicing für zeitkritische Anwendungen ... ermöglichen“ und deren „diskriminierungsfreie Ausgestaltung ... wettbewerbsrechtlich“ (FDP, 2021, 26) absichern wollen. In einer Gesamtschau lassen sich aus dem Programm telekommunikationspolitische Positionen (wettbewerbliche Marktordnung, auktionsbasierte Frequenzvergabe) der FDP klarer als bei den Sozialdemokraten entnehmen. 
Tabelle 1

Erwähnung medien- und digitalpolitischer Themen in Parteiprogrammen zur Bundestagswahl 2021

\begin{tabular}{|c|c|c|c|c|}
\hline \multirow{2}{*}{$\begin{array}{l}\text { Themenfeld } \\
\text { ÖRR }^{1} \text { und private Publikumsmedien }\end{array}$} & \multirow[t]{2}{*}{$\begin{array}{l}\text { Die } \\
\text { Linke }\end{array}$} & \multicolumn{3}{|c|}{$\begin{array}{c}\text { Die } \\
\text { SPD Grünen FDP }\end{array}$} \\
\hline & & & & \\
\hline Online Mediathek/Medienplattform & $\checkmark$ & $\checkmark$ & $\checkmark$ & - \\
\hline ÖRR-Konzentration Information /Bildung & $\checkmark$ & - & - & $\checkmark$ \\
\hline Urheberrecht & $\checkmark$ & - & $\checkmark$ & $\checkmark$ \\
\hline Leistungsschutzrecht Presseverleger & $\checkmark$ & - & - & $\checkmark$ \\
\hline \multicolumn{5}{|l|}{ Digitale Plattformkonzerne } \\
\hline Strukturelle Interventionen & $\checkmark$ & $\checkmark$ & $\checkmark$ & - \\
\hline Stärkung lokaler/regionaler Wettbewerber & $\checkmark$ & $\checkmark$ & $\checkmark$ & - \\
\hline Bezug zu Digital Market/Services Acts & - & $\checkmark$ & - & $\checkmark$ \\
\hline Bezug zu GWB & - & $\checkmark$ & $\checkmark$ & $\checkmark$ \\
\hline Automatisierte Abwehr Urheberrechtsverstöße & $\checkmark$ & - & $\checkmark$ & $\checkmark$ \\
\hline Abwehr Hassrede/Fehlinformationen & $\checkmark$ & $\checkmark$ & $\checkmark$ & $\checkmark$ \\
\hline Netzneutralität & $\checkmark$ & - & - & $\checkmark$ \\
\hline \multicolumn{5}{|l|}{ Ausbau Telekommunikationsnetze } \\
\hline Sektorremonopolisierung & $\checkmark$ & - & - & - \\
\hline Staatliche Ausbausubventionsprogramme & $\checkmark$ & - & $\checkmark$ & - \\
\hline Recht auf schnelles Internet & $\checkmark$ & $\checkmark$ & $\checkmark$ & - \\
\hline Liefertreue Breitbandanschluss & - & - & $\checkmark$ & - \\
\hline Gigabit-Gutscheine & - & - & - & $\checkmark$ \\
\hline Auktion als Regelverfahren Frequenzvergabe & - & - & $\checkmark$ & $\checkmark$ \\
\hline Lokales Roaming & - & - & $\checkmark$ & - \\
\hline
\end{tabular}

${ }^{1}$ ÖRR = öffentlich-rechtlicher Rundfunk.

Quelle: eigene Analysen.

\section{Zwischenfazit}

Beim Ausbau von Netzen zur elektronischen Kommunikation gehen die Programme der Linken und der SPD über wenige Schlagworte nicht hinaus. Grüne und FDP erwähnen einschlägige Fachthemen umfassender, bleiben aber hinter dem Status aktueller Rechtsentwicklungen und der wissenschaftlichen Diskussion zurück.

\section{Gesamtfazit und Ausblick}

Die politische Bedeutung der analysierten vier Wahlprogramme hängt von dem Mandatsanteil der Parteien nach der nächsten Bundestagswahl, ihrer Beteiligung an der Bundesregierung sowie von den Ressorts und sonstigen Spitzenstellen ab, die Parteimitglieder in dieser Regierung übernehmen werden. Es kann deshalb vermutet werden, dass dem Programm der Linken geringste und dem der
Grünen die größte Relevanz zukommen wird. Das Programm der Grünen zeichnet sich gegenüber den andern drei Absichtserklärungen durch eine relativ umfassende Benennung digital- und medienpolitischer Themen aus (vgl. Tabelle 1); es ist aber auch inhaltlich am wenigsten konturiert. Auf diese Weise maximiert die Partei ihre Chancen, keine Wählergruppe zu verschrecken und in der Folge durch eine Regierungsbeteiligung ihre politischen Vorstellungen realisieren zu können. Zudem spricht die Unbestimmtheit auf dem hier untersuchten Politikfeld dafür, dass die Grünen - und auch wohl die Linke, SPD und FDP - es allen Lippenbekenntnissen zur enormen Wichtigkeit der gesellschaftlichen Digitalisierung zum Trotz nicht als kritisch für ihre Wahlaussichten einstufen. Die Linke, SPD und FDP orientieren sich jeweils in ihren Wahlprogrammen bei der Digital- und Medienpolitik stärker als die Grünen am Markenkern der Partei. Allerdings werden auch in diesen drei Programmen Positionen nicht nachvollziehbar begründet. Bei wichtigen Aspekten sind die Vorstellungen veraltet, weil sie den Stand der Rechtssetzung und der Fachdebatten zu wenig beachten.

In Deutschland wird gegenwärtig, nicht zuletzt angesichts von Schwachstellen im Umgang mit der CoronaPandemie, von vielen beklagt, dass das Land bei der Digitalisierung international keine Spitzenposition einnimmt. Die Programme von vier Parteien für die Bundestagswahl 2021 vermitteln nicht den Eindruck, dass dieser Rückstand durch fachkundige Wettbewerbs- und Verbraucherschutzpolitik rasch aufgeholt werden dürfte. Es bleibt zu hoffen, dass diese Einschätzung zu pessimistisch ist, weil die Effekte von Wahlprogrammen auf das praktische Handeln der Bundesregierung und der Bundestagsparteien auch in der 20. Legislaturperiode gering ausfallen könnten.

\section{Literatur}

Buhrow, T. (2021), Wo die ARD im Jahr 2030 steht, Frankfurter Allgemeine Zeitung, 69, 23. März, 13

Bündnis 90/Die Grünen (2021), Deutschland. Alles ist drin. Programmentwurf zur Bundestagswahl 2021, https://cms.gruene.de/uploads/ documents/2021_Wahlprogrammentwurf.pdf (4. Mai 2021).

Bundesregierung (2021a), Entwurf eines Gesetzes zur Umsetzung der Richtlinie (EU) 2018/1972 des Europäischen Parlaments und des Rates vom 11. Dezember 2018 über den europäischen Kodex für die elektronische Kommunikation (Neufassung) und zur Modernisierung des Telekommunikationsrechts (Telekommunikationsmodernisierungsgesetz), Bundestagsdrucksache 19/26108, 25. Januar, https:// dip21.bundestag.de/dip21/btd/19/261/1926108.pdf (4. Mai 2021).

Bundesregierung (2021b), Entwurf eines Gesetzes zur Anpassung des Urheberrechts an die Erfordernisse des digitalen Binnenmarktes, Bundestagsdrucksache 19/27426 vom 9. März, http://dipbt.bundestag.de/dip21/btd/19/274/1927426.pdf (4. Mai 2021).

Clement, R., D. Schreiber, P. Bossauer und C. Pakusch (2019), InternetÖkonomie, 4. Aufl., Springer Gabler.

Deutscher Bundestag (2021), Beschlussempfehlung und Bericht des Ausschusses für Wirtschaft und Energie (9. Ausschuss), Bundes- 
tagsdrucksache 19/28865 vom 21. April, https://dip21.bundestag.de/ dip21/btd/19/288/1928865.pdf (4. Mai 2021).

Die Linke (2021), Zeit zu handeln: Für soziale Sicherheit, Frieden und Klimagerechtigkeit! Wahlprogramm der Partei DIE LINKE zur Bundestagswahl 2021, https://www.die-linke.de/fileadmin/download/wahlen2021/BTWP21_Entwurf_Vorsitzende.pdf (4. Mai 2021).

Europäische Kommission (2020a), Vorschlag für eine Verordnung des Europäischen Parlaments und des Rates über einen Binnenmarkt für digitale Dienste (Gesetz über digitale Dienste) und zur Änderung der Richtlinie 2000/31/EG, COM(2020) 825 final, https://eur-lex.europa. eu/legal-content/DE/TXT/PDF/?uri=CELEX:52020PC0825\&from=en (4. Mai 2021).

Europäische Kommission (2020b), Vorschlag für eine Verordnung des Europäischen Parlaments und des Rates über bestreitbare und faire Märkte im digitalen Sektor (Gesetz über digitale Märkte), COM(2020) 842 final, https://eur-lex.europa.eu/legal-content/DE/TXT/PDF/?uri= CELEX:52020PC0842\&from=en (4. Mai 2021)

FDP (2021), Programmentwurf der Freien Demokraten zur Bundestagswahl 2021 - Leitantrag des Bundesvorstandes zum 72. Bundesparteitag am 14.-16. Mai 2021 (vorbehaltlich der Überprüfung des Wortprotokolls), https://www.fdp.de/sites/default/files/uploads/2021/04/16/ programmentwurf-nie-gab-es-mehr-zu-tun-2.pdf (4. Mai 2021).

Gerpott, T. J. (2019), Artikel 17 der neuen EU-Urheberrechtsrichtlinie: Fluch oder Segen?, Multimedia und Recht, 22(7), 420-426.

Gerpott, T. J. (2020), Voucher für Glasfaseranschlüsse: Warum Bund und Länder auf sie verzichten sollten, Wirtschaftsdienst, 100(2), 128-132, https://www.wirtschaftsdienst.eu/inhalt/jahr/2020/heft/2/beitrag/ voucher-fuer-glasfaseranschluesse-warum-bund-und-laender-aufsie-verzichten-sollten.html (4. Mai 2021).

Gerpott, T. J. (2021a), Neue europäische Regeln für digitale Plattformen, Computer und Recht, 37(4), 255-263.

Gerpott, T. J. (2021b), Neue Pflichten für große Betreiber digitaler Plattformen - Vergleichende Analyse von Vorgaben auf Basis von § 19a GWB und des Vorschlags der Kommission für ein Gesetz über digitale Märkte, Neue Zeitschrift für Kartellrecht, 9(5), 273-279.
Gerpott, T. J. und T. Mikolas (2021), Zugang zu Daten großer Online-Plattformbetreiber nach der 10. GWB-Novelle. Computer und Recht, 37(2), 137-144.

Hartung, H. (2021), Alles online, alle unter einem Dach?, Frankfurter Allgemeine Zeitung, 98, 28. April, 13.

Krämer, J. und D. Schnurr (2021), Big data and digital markets contestability: Theory of harm and data access remedies, https://papers.ssrn.com/sol3/Delivery.cfm/SSRN_ID3789510_code702226. pdf?abstractid $=3789510 \&$ mirid $=1$ (4. Mai 2021).

Lettl, T. (2021), Der neue § 19a GWB, Wirtschaft und Wettbewerb, 67(4), 413-424.

Monopolkommission (2015), Wettbewerbspolitik: Herausforderung digitale Märkte, Sondergutachten 68, Juni, https://www.monopolkommission.de/images/PDF/SG/SG68/S68 volltext.pdf (4. Mai 2021).

Nagel, S. M. und K. Hillmer (2021), Die 10. GWB-Novelle - Update für die Missbrauchsaufsicht in der Digitalwirtschaft, Der Betrieb, 74(7), 327332.

Polley, R. und F. A. Konrad (2021), Der Digital Markets Act - Brüssels neues Regulierungskonzept für Digitale Märkte, Wirtschaft und Wettbewerb, 67(4), 198-206.

Schweda, M. und F. Schreitter (2021), Ran an die Datenschätze? Datenzugangsansprüche nach der 10. GWB-Novelle, Wirtschaft und Wettbewerb, 67(3), 145-154.

Seip, F. und M. Berberich (2021), Der Entwurf des Digital Markets Act, Gewerblicher Rechtsschutz und Urheberrecht Praxis im Immaterialgüterund Wettbewerbsrecht, 13(2), 44-47.

SPD (2021), Das Zukunftsprogramm - Wofür wir stehen. Was uns antreibt. Wonach wir streben, https://www.spd.de/fileadmin/Dokumente/Beschluesse/20210301_SPD_Zukunftsprogramm.pdf (4. Mai 2021).

Specht-Riemenschneider, L., S. Riemenschneider und R. Schneider (2020), Internetrecht, Springer.

Spindler, G. (2021), Der Vorschlag für ein neues Haftungsregime für Internetprovider - der EU-Digital Services Act (Teil 1), Gewerblicher Rechtsschutz und Urheberrecht, 123(4), 545-553.

Title: Digital and Media Policy in Party Programmes for the German Federal Parliamentary Elections in 2021 - How Clear and Convincing Are Parties' Messages?

Abstract: By late April 2021, four parties have published their programme for the German federal parliamentary elections (Bundestag) in September 2021. This article provides a critical comparative review of digital and media policy messages presented in these programmes. The Greens cover current topics in the areas of public service and commercial media large online platform providers and infrastructure roll out for electronic communications both most comprehensively and most vaguely. The digital and media policy statements of the left, social democratic and free democratic parties stick more closely to the respective party's brand. 